Gut and Liver, Vol. 12, No. 6, November 2018, pp. 655-663

\title{
Optimal Starting Age for Colorectal Cancer Screening in an Era of Increased Metabolic Unhealthiness: A Nationwide Korean Cross-Sectional Study
}

\author{
Yoon Jin Choi ${ }^{1, \star}$, Dong Ho Lee ${ }^{1,2}$, Kyung-Do Han ${ }^{3}$, Hyun Soo Kim ${ }^{1}$, Hyuk Yoon ${ }^{1}$, Cheol Min Shin ${ }^{1}$, Young Soo Park ${ }^{1,2}$, and \\ Nayoung $\mathrm{Kim}^{1,2}$ \\ ${ }^{1}$ Department of Internal Medicine, Seoul National University Bundang Hospital, Seongnam, ${ }^{2}$ Department of Internal Medicine and Liver \\ Research Institute, Seoul National University College of Medicine, Seoul, and ${ }^{3}$ Department of Biostatistics, College of Medicine, The Catholic \\ University of Korea, Seoul, Korea
}

Background/Aims: The association between metabolic syndrome and colorectal cancer (CRC) has been suggested as one of causes for the increasing incidence of CRC, particularly in younger age groups. The present study examined whether the current age threshold (50 years) for CRC screening in Korea requires modification when considering increased metabolic syndrome. Methods: We analyzed data from the National Health Insurance Corporation database, which covers $\sim 97 \%$ of the population in Korea. CRC risk was evaluated with stratification based on age and the presence/absence of relevant metabolic syndrome components (diabetes, dyslipidemia, and hypertension). Results: A total of 51,612,316 subjects enrolled during 2014 to 2015 were analyzed. Among them, 19.3\% had diabetes, hypertension, dyslipidemia, or some combination thereof. This population had a higher incidence of CRC than did those without these conditions, and this was more prominent in subjects $<40$ years of age. The optimal cutoff age for detecting CRC, based on the highest Youden index, was 45 years among individuals without diabetes, dyslipidemia, and hypertension. Individuals with at least one of these components of metabolic syndrome had the highest Youden index at 62 years old, but the value was only 0.2. Resetting the cutoff age from 50 years to 45 years achieved a $6 \%$ increase in sensitivity for CRC detection among the total population. Conclusions: Starting CRC screening earlier, namely, at 45 rather than at 50 years of age, may improve secondary prevention of CRC in Korea. (Gut Liver 2018;12:655-663)

Key Words: Colonic neoplasms; Screening; Youden index; Metabolic syndrome; Diabetes mellitus

\section{INTRODUCTION}

Colorectal cancer (CRC) is one of the most prevalent cancers in both men and women. Globally, 1.2 million new cases of CRC and 608,700 CRC-related deaths were estimated to have occurred in 2008. ${ }^{1}$ In Korea, CRC is the second most common cancers except for thyroid cancer, and the firth most common cause for cancer-related death. The incidence has sharply increased till 2010s, exceeding the incidence noted in the United States. ${ }^{2}$ The age-standardized incidence rate was 31.9 per 100,000 persons. ${ }^{2}$

Although CRC usually has an excellent prognosis when the disease is diagnosed at an early stage, ${ }^{3}$ only less than $40 \%$ of cases are diagnosed while the cancer is still localized. ${ }^{4}$ Therefore, early detection and intervention for preneoplastic and early neoplastic lesions are essential for secondary prevention of CRC.

In the general population, the risk of developing CRC rises sharply after the age of 40 years, and 90\% of cancers occur in persons aged 50 years or older. ${ }^{5}$ The Korean National Cancer Center recommends CRC screening for all individuals aged 50 to 75 years, which is in accordance with the guidelines of the U.S. Preventive Services Task Force. ${ }^{6}$ However, the prevalence of cancers of the colon and rectum appears to have increased among younger adults, ${ }^{7}$ possibly due to westernized dietary patterns. An increase of CRC could be attributable to increased obesity and metabolic syndrome $\mathrm{e}^{8-10}$ and the increasing trend has been expected to continue under prevalent westernized life style. However, few studies evaluated the effective screening age for early detecting of CRC in the era of increasing metabolic unhealthy status.

In this context, first, we analyzed the incidence of CRC for certain time period and compared the incidence rate of CRC

Correspondence to: Dong Ho Lee

Department of Internal Medicine, Seoul National University Bundang Hospital, 82 Gumi-ro 173beon-gil, Bundang-gu, Seongnam 13620, Korea

Tel: +82-31-787-7008, Fax: +82-31-787-4051, E-mail: dhljohn@yahoo.com

Received on November 16, 2017. Revised on February 16, 2018. Accepted on March 29, 2018. Published online September 21 , 2018

pISSN 1976-2283 eISSN 2005-1212 https://doi.org/10.5009/gnl17514

*Current affiliation of Yoon Jin Choi is Department of Internal Medicine, Korea University Kuro Hospital, Seoul, Korea.

@ This is an Open Access article distributed under the terms of the Creative Commons Attribution Non-Commercial License (http://creativecommons.org/licenses/by-nc/4.0) which permits unrestricted non-commercial use, distribution, and reproduction in any medium, provided the original work is properly cited. 
based on the absence or presence of diabetes mellitus (DM), hypertension, dyslipidemia, or some combination thereof. We aimed to investigate the optimal age for initiating CRC screening based on the presence/absence of metabolic syndrome components (DM, hypertension, and/or dyslipidemia) using Korean nationwide databases based on the Youden index.

\section{MATERIALS AND METHODS}

\section{Data source and study population}

Approximately 97\% of the Korean population is registered with the National Health Insurance Corporation (NHIC), ${ }^{11}$ which is a national insurer managed by the Korean government. Korean researchers can use the NHIC database with approval by the official review committee. Medical record data and cancer registry codes were evaluated for all individuals enrolled in the NHIC between 2008 and 2015. A considerable portion of NHIC subscribers were aged over 40 years and were thus semicompulsorily recommended to undergo a standardized medical examination at least biennially. Therefore, majority of adults take part in medical examination biennially. In order to obtain average values for CRC incidence and risk, the data were analyzed and presented in 2 consecutive years (Supplementary Fig. 1). Once an individual was diagnosed with CRC, the corresponding data were excluded from the evaluation of subsequent time periods. The primary endpoint was newly diagnosed CRC, which was defined using the International Classification of Diseases, 10th revision (ICD-10) codes C180-200 and D010-012. The CRC diagnosis was considered new when the patient had no such diagnosis during the preceding years. All patients with a previous malignancy were excluded. In order to evaluate the mortality of persons diagnosed with CRC during 2008-2009, they were fol- lowed up until 2015.

Standardized self-reporting questionnaires were completed at baseline for the following CRC risk factors, which were included as covariates in multivariable analyses: age (years), sex, residency (rural/urban), yearly income (lower quintile vs the remaining quintiles), alcohol intake (frequency: never or near abstinence, 2-3 times/month, 1-2 times/week, 3-4 times/week, and $\geq 5$ times/week; and amount: complete or near abstinence, <3, 6, 9, or 12 standard drinks of $10 \mathrm{~g}$ alcohol units per drink), cigarette smoking (never, former, and current), and physical activity level (low, moderate, or high). Since the study involved routinely collected data, informed consent was not specifically obtained for this study. This study was approved by the Institutional Review Board of Seoul National University Bundang Hospital (IRB number: X-1608/360-904).

\section{Definition of metabolic health status}

Metabolic syndrome consists of a cluster of risk factors for type 2 DM and cardiovascular diseases, and includes manifestations such as abdominal obesity, dysglycemia, raised blood pressure, and elevated triglyceride and cholesterol levels. ${ }^{8} \mathrm{Al}-$ though DM, hypertension, and dyslipidemia can be defined using claim codes instead of measured value, body mass index or waist circumference was not. Therefore, the three metabolic disease components (DM, hypertension, and dyslipidemia) were used to define metabolic health status in the present study. The presence of DM was defined as at least one claim per year for the prescription of antidiabetic medication (ICD-10 codes E1014). ${ }^{12}$ The presence of hypertension was defined as the presence of at least one claim per year for the prescription of an antihypertensive agent (ICD-10 codes I10-I15). Dyslipidemia was defined as at least one claim per year for the prescription of an

Table 1. Demographics of the Study Population

\begin{tabular}{lcccc}
\hline \multirow{2}{*}{ No. of enrollees } & \multicolumn{4}{c}{ Inspected time period ${ }^{*}$} \\
\cline { 2 - 5 } & $2008-2009(\mathrm{n}=50,087,762)$ & $2010-2011(\mathrm{n}=50,647,225)$ & $2012-2013(\mathrm{n}=51,116,467)$ & $2014-2015(\mathrm{n}=51,612,316)$ \\
\hline Age, yr & $37.0 \pm 20.3$ & $37.9 \pm 20.5$ & $38.8 \pm 20.8$ & $39.8 \pm 21.0$ \\
$<20$ & $11,739,523(23.4)$ & $11,346,409(22.4)$ & $1,085,1353(21.2)$ & $10,328,931(20.0)$ \\
$20-39$ & $15,672,345(31.3)$ & $15,198,420(30.0)$ & $14,869,691(29.1)$ & $14,702,049(28.5)$ \\
$40-59$ & $15,308,531(30.6)$ & $16,244,741(32.1)$ & $16,850,977(33.0)$ & $17,145,075(33.2)$ \\
$\geq 60$ & $7,367,363(14.7)$ & $7,857,655(15.5)$ & $8,544,446(16.7)$ & $9,436,261(18.3)$ \\
Male sex & $25,106,279(50.1)$ & $25,368,953(50.1)$ & $25,574,157(50.0)$ & $25,812,101(50.0)$ \\
Diabetes & $2,114,418(4.2)$ & $2,419,334(4.8)$ & $2,671,692(5.2)$ & $2,938,220(5.7)$ \\
Hypertension & $5,693,421(11.4)$ & $6,279,551(12.4)$ & $6,871,603(13.4)$ & $7,238,317(14.0)$ \\
Dyslipidemia & $2,879,621(5.8)$ & $3,630,336(7.2)$ & $4,412,718(8.6)$ & $5,397,685(10.5)$ \\
$\geq 1$ Metabolic components & $7,377,397(14.7)$ & $8,302,881(16.4)$ & $9,142,668(17.9)$ & $9,957,810(19.3)$ \\
Urban residency & $23,282,274(46.5)$ & $22,617,486(45.6)$ & $23,241,067(45.5)$ & $28,357,706(54.9)$ \\
\hline
\end{tabular}

Data are presented as the mean \pm SD or number $(\%)$.

*Because adults aged over 40 years undergo mostly biennial medical examinations for cancer screening by the Korean government, the incidence was calculated for time periods of 2 consecutive years. Once an individual was diagnosed with colorectal cancer, the corresponding data were excluded from the evaluation of subsequent time periods. 
antihyperlipidemic agent (ICD-10 codes E78). ${ }^{12}$

\section{Statistical analyses}

Data are presented as the mean \pm standard deviation for normally distributed, continuous variables and as proportions for categorical variables. Continuous variables were evaluated using Student t-test and nominal variables were evaluated using the chi-square test. CRC incidence rates were calculated by dividing the number of events by the person at risk. The predictive accuracy of age for CRC development was assessed by calculating the c-index based on a receiver operating characteristic (ROC) curve analysis. ROC curve is used to evaluate a marker's ability for classifying disease status, and the Youden index, the maximum potential effectiveness of a marker, is a common summary measure of the ROC curve. ${ }^{13}$ The cutoff value for age in the prediction of CRC was defined as the value with the highest Youden index (sensitivity+specificity-1). ${ }^{14}$

Statistical analyses were performed using SAS version 9.4 (SAS Institute, Cary, NC, USA) and R version 3.2.3 (The R Foundation for Statistical Computing, Vienna, Austria, https://www. r-project.org). A two-sided p-value of less than 0.05 was considered to indicate statistical significance.

Table 2. The Incidence Rate of Colorectal Cancer among the 2014 to 2015 Enrollees, Stratified by Gender, Age, Presence or Absence of Diabetes, Hypertension, and/or Dyslipidemia

\begin{tabular}{|c|c|c|c|c|c|c|c|c|c|c|}
\hline \multirow{2}{*}{ Age } & \multicolumn{3}{|c|}{ Total $(n=51,612,316)$} & \multicolumn{3}{|c|}{$\begin{array}{l}\text { Without diabetes, hypertension, } \\
\text { and dyslipidemia }(n=41,654,506)\end{array}$} & \multicolumn{3}{|c|}{$\begin{array}{l}\text { With diabetes, hypertension, } \\
\text { and/or dyslipidemia }(n=9,957,810)\end{array}$} & \multirow{2}{*}{$\operatorname{IRR}^{\dagger}$} \\
\hline & No. & Events & $\mathrm{IR}^{*}$ & No. & Events & $\mathrm{IR}^{*}$ & No. & Events & $\mathrm{IR}^{*}$ & \\
\hline \multicolumn{11}{|l|}{ Total, yr } \\
\hline$<20$ & $10,328,931$ & 44 & 0.43 & $1,031,4262$ & 43 & 0.42 & 14,669 & 1 & 6.82 & 16.35 \\
\hline $20-29$ & $6,881,163$ & 351 & 5.10 & $6,826,537$ & 339 & 4.97 & 54,626 & 12 & 21.97 & 4.42 \\
\hline $30-39$ & $7,820,886$ & 2,282 & 29.18 & $7,531,968$ & 2,132 & 28.31 & 288,918 & 150 & 51.92 & 1.83 \\
\hline $40-49$ & $8,855,679$ & 7,972 & 90.02 & $7,668,769$ & 6,670 & 86.98 & $1,186,910$ & 1,302 & 109.70 & 1.26 \\
\hline $50-59$ & $8,289,396$ & 17,862 & 215.48 & $5,558,063$ & 11,084 & 199.42 & $2,731,333$ & 6,778 & 248.16 & 1.24 \\
\hline $60-69$ & $5,002,872$ & 20,558 & 410.92 & $2,288,859$ & 8,888 & 388.32 & $2,714,013$ & 11,670 & 429.99 & 1.11 \\
\hline $70-79$ & $3,089,751$ & 19,092 & 617.91 & $1,004,401$ & 6,033 & 600.66 & $2,085,350$ & 13,059 & 626.23 & 1.04 \\
\hline$\geq 80$ & $1,343,638$ & 7,234 & 538.39 & 461,647 & 2,200 & 476.55 & 881,991 & 5,034 & 570.75 & 1.20 \\
\hline All & $51,612,316$ & 75,395 & 146.07 & $41,654,506$ & 37,389 & 90.00 & $9,957,810$ & 38,006 & 382.00 & 4.25 \\
\hline \multicolumn{11}{|l|}{ Male, yr } \\
\hline$<20$ & $5,352,436$ & 20 & 0.37 & $5,344,007$ & 19 & 0.36 & 8,429 & 1 & 11.86 & 33.37 \\
\hline 20-29 & $3,643,141$ & 141 & 3.87 & $3,605,904$ & 134 & 3.72 & 37,237 & 7 & 18.80 & 5.06 \\
\hline 30-39 & $4,017,889$ & 872 & 21.70 & $3,804,928$ & 791 & 20.79 & 212,961 & 81 & 38.04 & 1.83 \\
\hline $40-49$ & $4,495,684$ & 3,243 & 72.14 & $3,712,980$ & 2,498 & 67.28 & 782,704 & 745 & 95.18 & 1.41 \\
\hline $50-59$ & $4,162,460$ & 9,778 & 234.91 & $2,694,352$ & 5,725 & 212.48 & $1,468,108$ & 4,053 & 276.07 & 1.30 \\
\hline $60-69$ & $2,419,958$ & 12,888 & 532.57 & $1,127,057$ & 5,542 & 491.72 & $1,292,901$ & 7,346 & 568.18 & 1.16 \\
\hline 70-79 & $1,314,452$ & 11,226 & 854.04 & 481,458 & 3,791 & 787.4 & 832,994 & 7,435 & 892.56 & 1.13 \\
\hline$\geq 80$ & 406,081 & 3,391 & 835.06 & 152,006 & 1,075 & 707.21 & 254,075 & 2,316 & 911.54 & 1.29 \\
\hline All & $25,812,101$ & 41,559 & 161.01 & $20,922,692$ & 19,575 & 94.00 & $4,889,409$ & 21,984 & 450.00 & 4.81 \\
\hline \multicolumn{11}{|c|}{ Female, yr } \\
\hline$<20$ & $4,976,495$ & 24 & 0.48 & $4,970,255$ & 24 & 0.48 & 6,240 & 0 & - & - \\
\hline $20-29$ & $3,238,022$ & 210 & 6.49 & $3,220,633$ & 205 & 6.37 & 17,389 & 5 & 28.75 & 4.52 \\
\hline 30-39 & $3,802,997$ & 1,410 & 37.08 & $3,727,040$ & 1,341 & 36.98 & 75,957 & 69 & 90.84 & 2.52 \\
\hline $40-49$ & $4,359,995$ & 4,729 & 108.46 & $3,955,789$ & 4,172 & 105.47 & 404,206 & 557 & 137.80 & 1.31 \\
\hline $50-59$ & $4,126,936$ & 8,084 & 195.88 & $2,863,711$ & 5,359 & 187.14 & $1,263,225$ & 2,725 & 215.72 & 1.15 \\
\hline $60-69$ & $2,582,914$ & 7,670 & 296.95 & $1,161,802$ & 3,346 & 288.00 & $1,421,112$ & 4,324 & 304.27 & 1.06 \\
\hline 70-79 & $1,775,299$ & 7,866 & 443.08 & 522,943 & 2,242 & 428.73 & $1,252,356$ & 5,624 & 449.07 & 1.05 \\
\hline$\geq 80$ & 937,557 & 3,843 & 409.90 & 309,641 & 1,125 & 363.32 & 627,916 & 2,718 & 432.86 & 1.19 \\
\hline All & $25,800,215$ & 33,836 & 131.15 & $20,731,814$ & 17,814 & 86.00 & $5,068,401$ & 16,022 & 316.00 & 3.68 \\
\hline
\end{tabular}

IR, incidence rate; IRR, incidence rate ratio.

*Per 100,000 persons: because the evaluation was based on data from 2 consecutive years, the IR was approximately doubled; ${ }^{\dagger}$ Between subjects with/without metabolic components. 


\section{RESULTS}

\section{Characteristics of the study population}

Table 1 shows the baseline characteristics of the study population for each 2-year time period analyzed. In the present study, 50,087,762, 50,647,225, 51,116,467 and 51,612,316 of enrollees from 2008 to 2009, 2010 to 2011, 2012 to 2013, and 2014 to 2015, respectively, were analyzed. Approximately half of the total enrollees were men. The frequencies of DM, hypertension, and dyslipidemia increased significantly over time $(p<0.001$ for longitudinal trend). The proportions of having more than one component of DM, hypertension, or dyslipidemia were $14.8 \%, 16.5 \%, 18.0 \%$ and $19.3 \%$, respectively, for each time period. When the baseline characteristics according to sex were compared, same patterns were shown both in men and women (Supplementary Table 1). While higher proportion of enrollees were diagnosed with DM among men than women, those of hypertension or dyslipidemia were greater in women than men.

\section{CRC risk stratified by age group and metabolic healthy status}

In order to evaluate the CRC risk by age and metabolic healthy status, one time period (2014 to 2015) was analyzed as a representative. First, CRC incidences were 90.00 per 100,000 persons in the metabolic healthy group and 382.00 per 100,000 persons in the metabolic unhealthy group.

The CRC incidence among the 2014 to 2015 enrollees was stratified by age group in 10-year intervals and by the presence or absence of metabolic syndrome components (Table 2). Until 79 years of age, the total CRC incidence continuously increased with increasing age. The CRC incidence for the 2-year period of 2014 to 2015 was 215.48 cases per 100,000 persons for enrollees 50 to 59 years of age and 617.91 cases per 100,000 persons for enrollees 70 to 79 years of age. For persons aged over 80 years old, the IR was still higher than that of persons 50 to 59
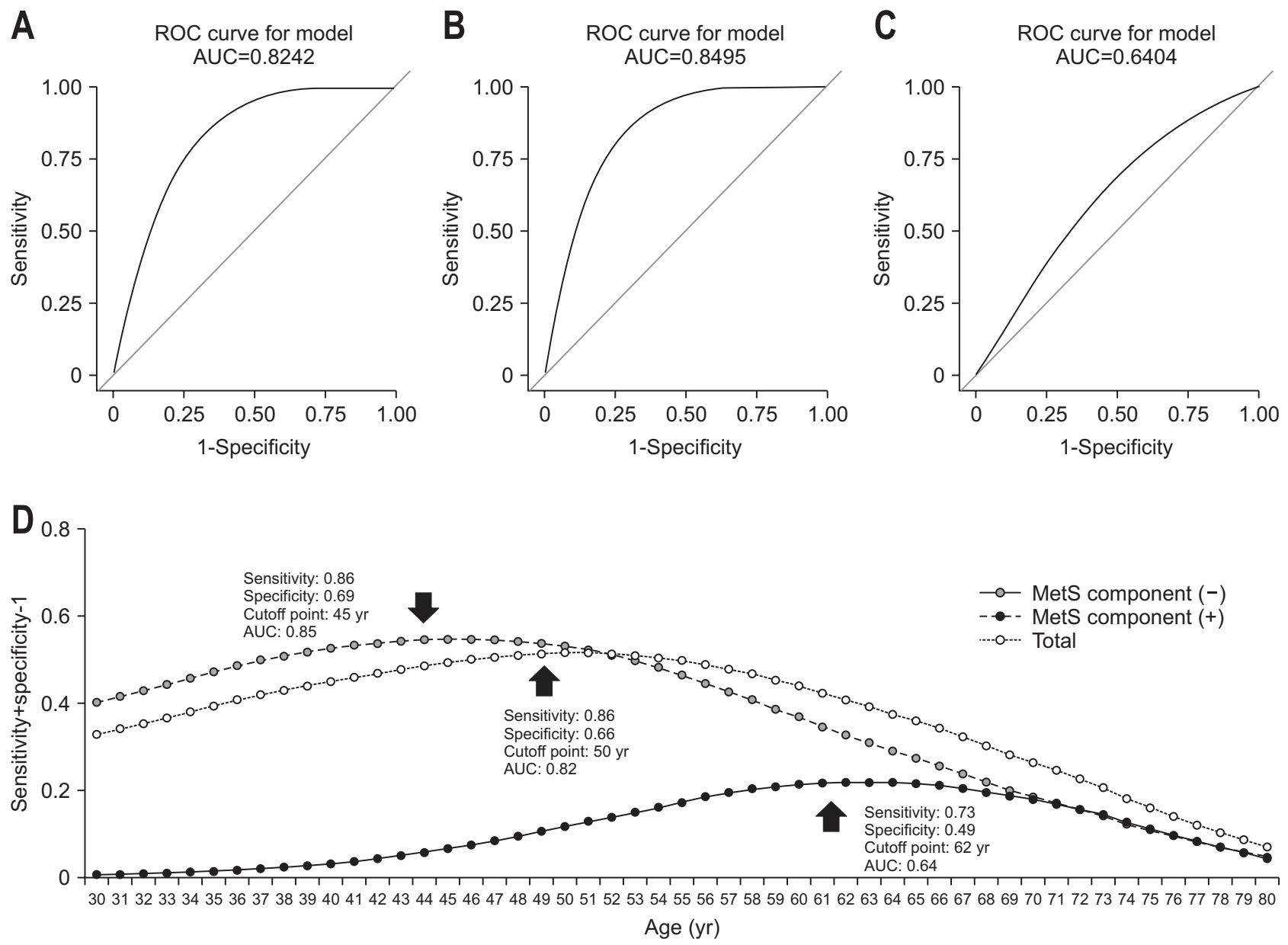

Fig. 1. Receiver operating characteristic (ROC) curves and Youden index for the prediction of colorectal cancer based on age. (A) Among the total population, the c-index was 0.82, and the cutoff age was 50 years, as determined by the highest Youden index (0.52). (B) Among individuals without any metabolic syndrome components, the cutoff age was 45 years (Youden index, 0.55). (C) Among individuals with one or more metabolic syndrome components, the cutoff age was 62 years, and the Youden index was 0.22. (D) The Youden index (sensitivity+specificity-1) for the above three groups is illustrated.

AUC, area under the ROC curve; MetS, metabolic syndrome. 
years of age (538.39 per 100,000 persons over 2 consecutive years).

Among enrollees during 2014 to 2015 , 9,957,810 subjects (19.3\%) had more than one of the metabolic disease components. This was consistent in all age groups, but more prominent for young adult groups, particularly for those under 40 years of age (Table 2). Among those aged 20 to 29 years, the incidence rate ratio was 4.42. Meanwhile, in those aged 30 to 39 years, there was a 1.83-fold higher incidence of CRC in individuals with metabolic syndrome component(s) compared to that for individuals without any metabolic component (Table 2).

\section{CRC risk stratified by gender and metabolic healthy status}

In order to evaluate the possible sex-based differences in the association between metabolic syndrome and CRC development, the incidence of CRC according to the presence or absence of the metabolic disease components were analyzed by gender (Table 2).

The metabolically unhealthy group which had more than one of the metabolic disease components showed a higher incidence rates of CRC compared with the metabolically healthy group both in men and women throughout the all age (men, 450 vs 94 ; women, 316 vs 86 ). The incidence rate ratios were generally greater in men than in women except for 30 s.

\section{Cutoff age for the prediction of CRC}

Fig. 1 shows the ROC curve analysis for the best age-based prediction of CRC diagnosis for the enrollees during the period 2014 to 2015. For all enrollees, the c-index for age was 0.82 , and the cutoff value was 50 years. The sensitivity, specificity, and Youden index for the prediction of CRC at different cutoff ages are shown in Table 3. In addition, the cutoff values and c-index for age were stratified by the presence or absence of metabolic components (DM, hypertension, and/or dyslipidemia) (Table 3). The best cutoff age for CRC detection among individuals without any of metabolic component was 45 years, while that for individuals with more than one metabolic component was 62 years. The c-index was lower in individuals with DM, hypertension, and/or dyslipidemia compared to that in individuals with no metabolic component (0.85 vs 0.64) (Fig. 1).

Table 3. The Youden Index for the Detection of Colorectal Cancer According to Age, Evaluated among 2014 to 2015 Enrollees

\begin{tabular}{|c|c|c|c|c|c|c|c|c|c|c|c|}
\hline \multicolumn{4}{|c|}{ Total $(n=51,612,316)$} & \multicolumn{4}{|c|}{$\begin{array}{l}\text { Without diabetes, hypertension, } \\
\text { and dyslipidemia }(n=41,654,506)\end{array}$} & \multicolumn{4}{|c|}{$\begin{array}{l}\text { With diabetes, hypertension, } \\
\text { and/or dyslipidemia }(n=9,957,810)\end{array}$} \\
\hline $\begin{array}{l}\text { Cutoff } \\
\text { age, yr }\end{array}$ & SENS & SPEC & YI & $\begin{array}{l}\text { Cutoff } \\
\text { age, yr }\end{array}$ & SENS & SPEC & YI & $\begin{array}{l}\text { Cutoff } \\
\text { age, yr }\end{array}$ & SENS & SPEC & YI \\
\hline 41 & 0.958 & 0.501 & 0.459 & 36 & 0.965 & 0.522 & 0.486 & 50 & 0.961 & 0.156 & 0.117 \\
\hline 42 & 0.951 & 0.518 & 0.468 & 37 & 0.957 & 0.541 & 0.498 & 51 & 0.953 & 0.176 & 0.129 \\
\hline 43 & 0.942 & 0.535 & 0.478 & 38 & 0.950 & 0.558 & 0.508 & 52 & 0.942 & 0.197 & 0.139 \\
\hline 44 & 0.933 & 0.553 & 0.486 & 39 & 0.942 & 0.576 & 0.517 & 53 & 0.931 & 0.219 & 0.149 \\
\hline 45 & 0.922 & 0.571 & 0.493 & 40 & 0.933 & 0.593 & 0.526 & 54 & 0.915 & 0.245 & 0.160 \\
\hline 46 & 0.911 & 0.589 & 0.500 & 41 & 0.921 & 0.610 & 0.532 & 55 & 0.898 & 0.274 & 0.172 \\
\hline 47 & 0.899 & 0.607 & 0.506 & 42 & 0.907 & 0.629 & 0.537 & 56 & 0.880 & 0.306 & 0.186 \\
\hline 48 & 0.886 & 0.625 & 0.511 & 43 & 0.893 & 0.649 & 0.541 & 57 & 0.857 & 0.338 & 0.195 \\
\hline 49 & 0.872 & 0.641 & 0.514 & 44 & 0.877 & 0.668 & 0.545 & 58 & 0.835 & 0.368 & 0.203 \\
\hline $50^{*}$ & 0.859 & 0.657 & $0.516^{*}$ & $45^{*}$ & 0.858 & 0.688 & $0.546^{*}$ & 59 & 0.808 & 0.400 & 0.208 \\
\hline 51 & 0.842 & 0.674 & 0.516 & 46 & 0.839 & 0.707 & 0.546 & 60 & 0.783 & 0.430 & 0.213 \\
\hline 52 & 0.823 & 0.690 & 0.513 & 47 & 0.819 & 0.726 & 0.545 & 61 & 0.753 & 0.464 & 0.216 \\
\hline 53 & 0.804 & 0.705 & 0.510 & 48 & 0.797 & 0.745 & 0.542 & $62^{*}$ & 0.726 & 0.493 & $0.219^{*}$ \\
\hline 54 & 0.781 & 0.723 & 0.504 & 49 & 0.776 & 0.761 & 0.537 & 63 & 0.697 & 0.520 & 0.218 \\
\hline 55 & 0.757 & 0.740 & 0.497 & 50 & 0.754 & 0.777 & 0.531 & 64 & 0.667 & 0.551 & 0.218 \\
\hline 56 & 0.731 & 0.758 & 0.489 & 51 & 0.729 & 0.793 & 0.522 & 65 & 0.641 & 0.574 & 0.215 \\
\hline 57 & 0.704 & 0.774 & 0.478 & 52 & 0.702 & 0.808 & 0.510 & 66 & 0.612 & 0.600 & 0.211 \\
\hline 58 & 0.678 & 0.790 & 0.467 & 53 & 0.676 & 0.822 & 0.497 & 67 & 0.578 & 0.626 & 0.204 \\
\hline 59 & 0.648 & 0.804 & 0.452 & 54 & 0.645 & 0.836 & 0.482 & 68 & 0.542 & 0.653 & 0.195 \\
\hline 60 & 0.622 & 0.818 & 0.440 & 55 & 0.614 & 0.851 & 0.465 & 69 & 0.507 & 0.681 & 0.188 \\
\hline
\end{tabular}

Without metabolic components: absence of diabetes, hypertension, and dyslipidemia; with metabolic components: presence of diabetes, hypertension, or dyslipidemia.

SENS, sensitivity; SPEC, specificity; YI, Youden index=sensitivity+specificity-1.

*Age with the highest summation of sensitivity and specificity. 
Table 4. Mortality Rate and Hazard Ratio among Patients Diagnosed with Colorectal Cancer during 2008 to 2009 (Observed until 2015)

\begin{tabular}{|c|c|c|c|c|c|c|}
\hline & \multicolumn{2}{|c|}{ Total } & \multicolumn{2}{|c|}{ Male } & \multicolumn{2}{|c|}{ Female } \\
\hline & $\mathrm{MR}^{*}$ & $\mathrm{HR}(95 \% \mathrm{CI})^{\dagger}$ & $\mathrm{MR}^{*}$ & $\mathrm{HR}(95 \% \mathrm{CI})^{\dagger}$ & $\mathrm{MR}^{*}$ & $\mathrm{HR}(95 \% \mathrm{CI})^{\dagger}$ \\
\hline \multicolumn{7}{|l|}{ Age, yr } \\
\hline$<20$ & 48.78 & $1.53(0.81-2.87)$ & 72.51 & $2.40(1.12-5.14)$ & 27.66 & $0.82(0.26-2.57)$ \\
\hline $20-29$ & 45.85 & $1.46(1.12-1.90)$ & 53.53 & $1.77(1.22-2.58)$ & 39.94 & $1.20(0.82-1.74)$ \\
\hline $30-39$ & 31.62 & 1 (reference) & 29.96 & 1 (reference) & 33.20 & 1 (reference) \\
\hline $40-49$ & 34.09 & $1.07(0.94-1.22)$ & 35.78 & $1.19(0.99-1.44)$ & 32.30 & $0.97(0.81-1.16)$ \\
\hline $50-59$ & 34.95 & $1.08(0.96-1.22)$ & 38.71 & $1.29(1.08-1.54)$ & 29.59 & $0.89(0.75-1.06)$ \\
\hline $60-69$ & 43.45 & $1.34(1.19-1.50)$ & 47.17 & $1.57(1.32-1.86)$ & 37.14 & $1.12(0.95-1.32)$ \\
\hline $70-79$ & 74.35 & $2.28(2.03-2.57)$ & 81.25 & $2.68(2.26-3.18)$ & 64.75 & $1.93(1.64-2.27)$ \\
\hline$\geq 80$ & 164.09 & $5.03(4.46-5.67)$ & 167.74 & $5.42(4.54-6.47)$ & 160.96 & $4.64(3.93-5.47)$ \\
\hline All & 53.51 & & 56.68 & & 49.26 & \\
\hline \multicolumn{7}{|l|}{ DM } \\
\hline No & 50.04 & 1 (reference) & 53.06 & 1 (reference) & 46.02 & 1 (reference) \\
\hline Yes & 71.60 & $1.23(1.19-1.28)$ & 73.22 & $1.24(1.19-1.30)$ & 68.85 & $1.21(1.14-1.29)$ \\
\hline \multicolumn{7}{|l|}{ HTN } \\
\hline No & 48.97 & 1 (reference) & 53.13 & 1 (reference) & 43.28 & 1 (reference) \\
\hline Yes & 61.42 & $0.95(0.92-0.99)$ & 62.48 & $0.94(0.91-0.98)$ & 59.92 & $0.97(0.92-1.02)$ \\
\hline \multicolumn{7}{|l|}{ DysL } \\
\hline No & 54.97 & 1 (reference) & 57.98 & 1 (reference) & 50.66 & 1 (reference) \\
\hline Yes & 45.51 & $0.75(0.72-0.79)$ & 48.19 & $0.77(0.73-0.82)$ & 42.36 & $0.73(0.68-0.78)$ \\
\hline \multicolumn{7}{|c|}{ Components of MS } \\
\hline No & 47.80 & 1 (reference) & 51.71 & 1 (reference) & 42.52 & 1 (reference) \\
\hline$\geq 1$ & 60.23 & $0.97(0.94-1.00)$ & 62.17 & $0.98(0.94-1.02)$ & 27.66 & $0.95(0.91-1.00)$ \\
\hline
\end{tabular}

MR, mortality rate; HR, hazard ratio; CI, confidence interval; DM, diabetes mellitus; HTN, hypertension; DysL, dyslipidemia; MS, metabolic syndrome.

*Per 1,000 person-years; ${ }^{\dagger}$ Age, sex-adjusted.

When the sensitivity, specificity, and Youden index for the prediction of CRC at different cutoff ages were analyzed according to gender (Supplementary Fig. 2), the best cutoff age for CRC was 51 for men and 47 for women. The ROC curves were presented according to sex. Values of area under the curve were 0.86 among men and 0.79 among women.

\section{CRC patient mortality stratified according to age and metabolic components}

Table 4 shows the unadjusted rate for all-cause mortality of patients diagnosed with CRC, stratified according to baseline age. The overall mortality rate was 53.51 events per 1,000 person-years among enrollees with CRC. The rate ratios for allcause mortality were higher among older enrollees than among younger enrollees. However, the mortality rate among enrollees aged $<29$ years old was higher compared to that among individuals aged 30 to 69 years.

The unadjusted rates of all-cause mortality according to the presence or absence of metabolic components were calculated (Table 4). The rate ratio for all-cause mortality was higher among individuals with DM than among non-diabetic individuals (hazard ratio [HR], 1.24; 95\% confidence interval [CI], 1.19 to 1.30); however, dyslipidemia showed a protective effect (HR, 0.77 ; 95\% CI, 0.73 to 0.82 ). Same patterns were shown in men and women, but mortality rate was greater among men than among women (56.58 vs 49.26).

\section{DISCUSSION}

In the present nationwide study, which involved a large cohort of Korean individuals, we presented that resetting the CRC screening threshold to 45 years of age may improve the chance of identifying CRC in asymptomatic individuals. Individuals with DM, dyslipidemia, and/or hypertension had a higher incidence of CRC compared to their counterparts and this excess incidence was more prominent under 40 years of age. All-cause mortality of CRC was higher in subjects with DM than in nonDM subjects.

In Korea, the government provides annual fecal occult blood tests (FOBTs) for all adults over 50 years of age in order to 
promote early detection of lesions in asymptomatic subjects in accordance with U.S. Preventive Services Task Force. ${ }^{6}$ However, considering the rapidly increasing prevalence of CRC and the previously undocumented high incidence among young adults, ${ }^{7}$ earlier colorectal screening may be more efficient. In the present study, the incidence of CRC among young adults was much higher in 2014 to 2015 than in 2008 to 2009 (17.91 per 100,000 persons vs 11.36 per 100,000 persons) (Supplementary Table 2). This highlights the potential need of expanding the age criteria to include younger adults in the screening program.

Notably, the present study showed that persons with metabolic components and who were younger than 40 years of age had an excessive risk of CRC compared to metabolically healthy individuals. Similarly, the increased CRC risk in DM patients has been reported to be even higher in those who were younger than 55 years of age (HR, 2.0; 95\% CI, 1.0 to 3.8)..$^{15}$ Given that persons with metabolic syndrome have a higher incidence of CRC and a higher mortality rate, ${ }^{16}$ CRC screening in this subgroup will become an important issue.

Moreover, this study showed that the mortality of individuals younger than 30 years of age was higher compared to that in individuals aged 40 to 50 years in accordance with the study in the United States. ${ }^{7}$ Majority of young-onset CRC patients had advanced stage of the disease has been reported. ${ }^{17}$ Although the worse outcome of CRC among young adults could result from more aggressive biological feature or a lack of screening, it may be also related with increased prevalence of metabolic syndrome and obesity that involve the development of CRC in young adults. ${ }^{18,19}$ It has been reported that metabolic syndrome or DM is associated with not only an increased risk for $\mathrm{CRC}^{20-23}$ but all-cause and CRC-specific mortality. ${ }^{24-27}$ The present study also showed the increased mortality in CRC patients with DM. However, dyslipidemia was associated with a lower mortality rate might be due to the anti-CRC properties of statin. ${ }^{28}$

Although the present study showed that the sum of sensitivity and specificity was the highest at 62 years of age among individuals with metabolic syndrome components, this does not simply indicate that persons with DM, hypertension, or dyslipidemia were more likely to have CRC later than counterparts. Rather, this result implies there may be more significant factors for the CRC carcinogenesis among metabolic unhealthy individuals than age for. The area under the ROC curve (AUC) at age of 62 among metabolic unhealthy persons was as low as 0.64 . This supports that the age-based model could not detect CRC efficiently in persons with metabolic syndrome components. In line with this, the value of AUC has been constantly decreased in a situation of increased prevalence of metabolic syndrome (Supplementary Table 3). After excluding individuals with metabolic components, who account for $20 \%$ of total population, the cutoff age for the best detection of CRC dropped to 45 years old. The relatively minority put back the cutoff age when it was combined to metabolic healthy individuals (Fig. 1).
From all these aspects, resetting the cutoff age from 50 to 45 years achieved a $6 \%$ increment in sensitivity for CRC detection among the entire population is worthy of notice. In accordance with our data, European guidelines recommend annual or biennial FOBTs for asymptomatic adults from 45 years old. ${ }^{29}$ The Korean CRC screening guideline committee recently suggested decreasing the starting age of CRC screening by 5 years (from the previous recommendation of 50 years) in asymptomatic individuals. ${ }^{30}$ However, updated guideline for CRC screening by a U.S. Multi-Society Task Force still adheres to the start of CRC screening at the age of 50 years old. ${ }^{31}$ Young-onset CRC may require another strategy for risk stratification. ${ }^{32,33}$

Another issue about the metabolic healthy and the development of CRC is the gender-based difference. Several studies have indicated that there are gender-associated differences in the carcinogenesis of CRC. Proximal colon cancer is more often in females than in males. Man is associated with a higher CRC incidence and worse prognosis. ${ }^{7}$ A recent study in Korea showed that high body mass index was more associated with increased risk of CRC among men compared to women. ${ }^{34}$ In accordance with this result, the risk of CRC in metabolically unhealthy group compared with the metabolically healthy group was much higher among men than women (Table 2). On the other hand, guideline for CRC screening does not provide genderspecific recommendation. Although this study showed that the best cutoff age for CRC for women was 4 years earlier than that of men, this needs a cautious interpretation due to the lower value of AUC among women. A recent study presented that the incidence of CRC in populations over 80 years old was higher in women than that in men, but that of early onset CRC $(<50$ years old) was almost same in both sexes. ${ }^{7}$ According to the present study, the incidence of CRC in individuals 20 to 39 years of age was higher among women than among men (Supplementary Table 2) and this could put cutoff age forward in women. However, whether sex-associated differences in incidence of CRC exist among young adults ( $<40$ years old) needs further study because a study from Pakistan have reported male predominance. ${ }^{17}$ Moreover, analyses of genetic predispositions and may be more important to detect young-onset CRC. ${ }^{35}$

One of the strengths of the present study includes its use of a large, nationwide database. The limitations of the present study include the lack of data on waist circumference or waist-hip ratio allowing the evaluation of visceral obesity, one of the components of metabolic syndrome. While DM (a key factor), hypertension, and dyslipidemia are easily determined using claim data, visceral fat is not. Moreover, family history of CRC as well as the histological type, location and stage could not be evaluated. The results in the present study should be interpreted cautiously because we could not assess the effect of obesity which has associations between both CRC and metabolic syndrome.

The National Cancer Screening Program in Korea could start CRC screening at 45 years of age in increasing CRC among 
adults $<50$ years old. Moreover, given the higher incidence of CRC in individuals with DM, dyslipidemia, and/or hypertension, CRC screening in this group may be applied using the same cutoff for age as that used for the general population. Further study for validation of the effectiveness of screening in persons $<50$ years of age in persons with metabolic syndrome or DM is required.

\section{CONFLICTS OF INTEREST}

No potential conflict of interest relevant to this article was reported.

\section{ACKNOWLEDGEMENTS}

This work was supported by a grant from the National Research Foundation (NRF) of Korea to the Global Core Research Center (GCRC) funded by the Korean government (Ministry of Science, ICT and Future Planning) (No. 2011-0030001).

Author contribution: YJC analyzed the data and drafted the article; DHL designed the study and edited the manuscript; KDH performed data handling and statistical analysis; HSK, HY, CMS, and YSP provided statistical support and analyzed the questionnaires; NK provided advice on the study design. All authors have read and approved the manuscript.

\section{REFERENCES}

1. Jemal A, Bray F, Center MM, Ferlay J, Ward E, Forman D. Global cancer statistics. CA Cancer J Clin 2011;61:69-90.

2. Jung KW, Won YJ, Oh CM, et al. Cancer statistics in Korea: incidence, mortality, survival, and prevalence in 2014. Cancer Res Treat 2017;49:292-305.

3. Jemal A, Clegg LX, Ward E, et al. Annual report to the nation on the status of cancer, 1975-2001, with a special feature regarding survival. Cancer 2004;101:3-27.

4. American Cancer Society. Colorectal cancer facts \& figures 20142016 [Internet]. Atlanta: American Cancer Society [cited 2017 May 6]. Available from: http://www.cancer.org/research/cancerfacts-statistics/colorectal-cancer-facts-figures.html.

5. Haggar FA, Boushey RP. Colorectal cancer epidemiology: incidence, mortality, survival, and risk factors. Clin Colon Rectal Surg 2009;22:191-197.

6. U.S. Preventive Services Task Force. Screening for colorectal cancer: U.S. Preventive Services Task Force recommendation statement. Ann Intern Med 2008;149:627-637.

7. Siegel RL, Miller KD, Fedewa SA, et al. Colorectal cancer statistics, 2017. CA Cancer J Clin 2017;67:177-193.

8. Alberti KG, Eckel RH, Grundy SM, et al. Harmonizing the metabolic syndrome. Circulation 2009;120:1640-1645.

9. Moghaddam AA, Woodward M, Huxley R. Obesity and risk of colorectal cancer: a meta-analysis of 31 studies with 70,000 events. Cancer Epidemiol Biomarkers Prev 2007;16:2533-2547.

10. Esposito K, Chiodini P, Colao A, Lenzi A, Giugliano D. Metabolic syndrome and risk of cancer: a systematic review and metaanalysis. Diabetes Care 2012;35:2402-2411.

11. Lee J, Lee JS, Park SH, Shin SA, Kim K. Cohort profile: the National Health Insurance Service-National Sample Cohort (NHISNSC), South Korea. Int J Epidemiol 2017;46:e15.

12. Yang HK, Han K, Kwon HS, et al. Obesity, metabolic health, and mortality in adults: a nationwide population-based study in Korea. Sci Rep 2016;6:30329.

13. Ruopp MD, Perkins NJ, Whitcomb BW, Schisterman EF. Youden index and optimal cut-point estimated from observations affected by a lower limit of detection. Biom J 2008;50:419-430.

14. Youden WJ. Index for rating diagnostic tests. Cancer 1950;3:3235.

15. de Kort S, Masclee AA, Sanduleanu S, et al. Higher risk of colorectal cancer in patients with newly diagnosed diabetes mellitus before the age of colorectal cancer screening initiation. Sci Rep 2017;7:46527.

16. Ranc K, Jørgensen ME, Friis S, Carstensen B. Mortality after cancer among patients with diabetes mellitus: effect of diabetes duration and treatment. Diabetologia 2014;57:927-934.

17. Riaz R, Masood N, Benish A. Red flag symptoms: detailed account of clinicopathological features in young-onset colorectal cancer. Intest Res 2017;15:203-207.

18. Ginter E, Simko V. Type 2 diabetes mellitus, pandemic in 21st century. Adv Exp Med Biol 2012;771:42-50.

19. Ahrens W, Moreno LA, Mårild S, et al. Metabolic syndrome in young children: definitions and results of the IDEFICS study. Int J Obes (Lond) 2014;38 Suppl 2:S4-S14.

20. Pelucchi C, Negri E, Talamini R, et al. Metabolic syndrome is associated with colorectal cancer in men. Eur J Cancer 2010;46:18661872.

21. Huang CW, Sun LC, Shih YL, et al. The impact on clinical outcome of high prevalence of diabetes mellitus in Taiwanese patients with colorectal cancer. World J Surg Oncol 2012;10:76.

22. Yang MH, Rampal S, Sung J, et al. The association of serum lipids with colorectal adenomas. Am J Gastroenterol 2013;108:833-841.

23. Yao X, Tian Z. Dyslipidemia and colorectal cancer risk: a metaanalysis of prospective studies. Cancer Causes Control 2015;26: 257-268.

24. Stein KB, Snyder CF, Barone BB, et al. Colorectal cancer outcomes, recurrence, and complications in persons with and without diabetes mellitus: a systematic review and meta-analysis. Dig Dis Sci 2010;55:1839-1851.

25. Mills KT, Bellows CF, Hoffman AE, Kelly TN, Gagliardi G. Diabetes mellitus and colorectal cancer prognosis: a meta-analysis. Dis Colon Rectum 2013;56:1304-1319.

26. Chen KH, Shao YY, Lin ZZ, et al. Type 2 diabetes mellitus is associated with increased mortality in Chinese patients receiving curative surgery for colon cancer. Oncologist 2014;19:951-958.

27. Yap R, Wilkins S, Staples M, Oliva K, McMurrick PJ. The effect of 
diabetes on the perioperative outcomes of colorectal cancer surgery patients. PLoS One 2016;11:e0167271.

28. Poynter JN, Gruber SB, Higgins PD, et al. Statins and the risk of colorectal cancer. N Engl J Med 2005;352:2184-2192.

29. European Colorectal Cancer Screening Guidelines Working Group, von Karsa L, Patnick J, et al. European guidelines for quality assurance in colorectal cancer screening and diagnosis: overview and introduction to the full supplement publication. Endoscopy 2013;45:51-59.

30. Sohn DK, Kim MJ, Park Y, et al. The Korean guideline for colorectal cancer screening. J Korean Med Assoc 2015;58:420-432.

31. Rex DK, Boland CR, Dominitz JA, et al. Colorectal cancer screening: recommendations for physicians and patients from the U.S. Multi-Society Task Force on colorectal cancer. Gastroenterology
2017;153:307-323.

32. Jung YS. Is colorectal cancer screening necessary before 50 years of age? Intest Res 2017;15:550-551.

33. Jung YS, Park CH, Kim NH, Park JH, Park DI, Sohn CI. Colorectal cancer screening with the fecal immunochemical test in persons aged 30 to 49 years: focusing on the age for commencing screening. Gastrointest Endosc 2017;86:892-899.

34. Shin CM, Han K, Lee DH, et al. Association among obesity, metabolic health, and the risk for colorectal cancer in the general population in Korea using the National Health Insurance ServiceNational Sample Cohort. Dis Colon Rectum 2017;60:1192-1200.

35. Stoffel EM, Koeppe E, Everett J, et al. Germline genetic features of young individuals with colorectal cancer. Gastroenterology 2018; 154:897-905.e1. 